\title{
Synthesis and reactivity of BINEPINE-based chiral Fe(II) PNP pincer complexes
}

\author{
Christian Schröder-Holzhacker $^{1} \cdot$ Nikolaus Gorgas $^{1} \cdot$ Berthold Stöger $^{2} \cdot$ Karl Kirchner $^{1}$
}

Received: 8 January 2016/Accepted: 11 February 2016/Published online: 21 March 2016

(C) The Author(s) 2016. This article is published with open access at Springerlink.com

\begin{abstract}
A new asymmetric chiral PNP ligand based on the 2,6-diaminopyridine scaffold featuring a $R$-BINEPINE moiety was prepared. Treatment of anhydrous $\mathrm{FeX}_{2}$ $(\mathrm{X}=\mathrm{Cl}, \mathrm{Br})$ with 1 equiv of PNP-iPr,BIN at room temperature afforded the coordinatively unsaturated paramagnetic complexes $\left[\mathrm{Fe}(\mathrm{PNP}-i \mathrm{Pr}, \mathrm{BIN}) \mathrm{X}_{2}\right]$. The structure of $\left[\mathrm{Fe}(\mathrm{PNP}-i \mathrm{Pr}, \mathrm{BIN}) \mathrm{Cl}_{2}\right]$ is described. Both complexes react readily with the strong $\pi$-acceptor ligand $\mathrm{CO}$ in solution to afford selectively the diamagnetic complexes trans-[Fe(PNP-iPr,BIN $\left.)(\mathrm{CO}) \mathrm{X}_{2}\right]$ in quantitative yield. Due the lability of the $\mathrm{CO}$ ligand, these complexes are only stable under a $\mathrm{CO}$ atmosphere and isolation in pure form was not possible. The preparation of the carbonyl hydride complex $[\mathrm{Fe}(\mathrm{PNP}-i \mathrm{Pr}, \mathrm{BIN})(\mathrm{H})(\mathrm{CO}) \mathrm{Br}]$ was achieved albeit in low yields via a one pot procedure by treatment of [Fe(PNP-iPr,BINEP) $\mathrm{Br}_{2}$ ] with $\mathrm{CO}$ and subsequent reaction with $\mathrm{Na}\left[\mathrm{HBEt}_{3}\right]$. This complex was obtained as an inseparable mixture of two diastereomers in a ca. 1:1 ratio and was tested as catalyst for the hydrogenation of ketones. The catalyst showed acceptable activity under mild conditions (5 bar $\mathrm{H}_{2}$, room temperature) with yields up to $>99 \%$ within $18 \mathrm{~h}$.
\end{abstract}

Electronic supplementary material The online version of this article (doi:10.1007/s00706-016-1706-x) contains supplementary material, which is available to authorized users.

Karl Kirchner

kkirch@mail.tuwien.ac.at

1 Institute of Applied Synthetic Chemistry, Vienna University of Technology, Getreidemarkt 9/163-OC, 1060 Vienna, Austria

2 Institute of Chemical Technologies and Analytics, Vienna University of Technology, Getreidemarkt 9, 1060 Vienna, Austria

\section{Graphical abstract}

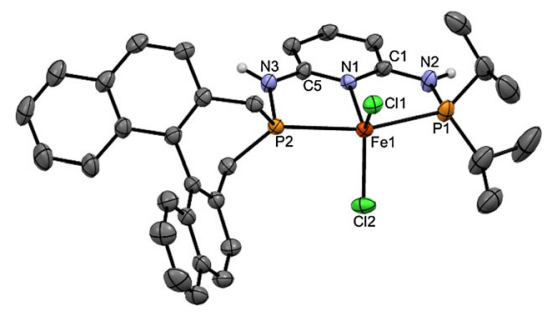

Keywords Iron · Pincer ligands - Chiral phosphines · Carbon monoxide

\section{Introduction}

In view of concerns regarding economy, environment, and sustainable energy, there is a constant need for the discovery of new catalytic reactions. A process we are interested in is the catalytic hydrogenation of polar multiple bonds via molecular hydrogen. This plays a significant role in modern synthetic organic chemistry and is excellently performed by many transition metal hydride complexes containing noble metals such as ruthenium, rhodium, or iridium [1-5]. The limited availability of precious metals, their high price, and their toxicity lowers the attractivity of these metals in the future and more economical and environmentally friendly alternatives have to be found which are in line with green chemistry guidelines. In this respect, the preparation of well-defined iron-based hydride catalysts of comparable or even higher activity is desirable [6-12]. Iron is the most abundant transition metal in the earth crust, and ubiquitously 


\section{Scheme 1}

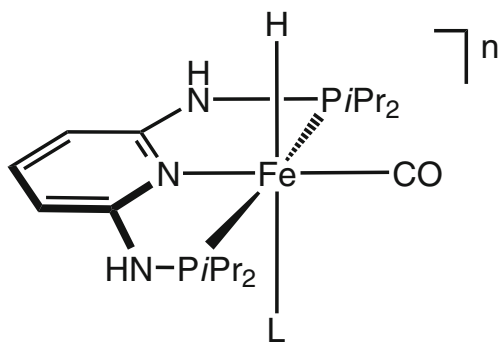

$\mathrm{L}=\mathrm{Br}^{-}, \mathrm{CH}_{3} \mathrm{CN}, \mathrm{BH}_{4}^{-}$

available. Accordingly, it is not surprising that the field of iron catalyzed hydrogenations of polar multiple bonds is rapidly evolving as shown by several recent examples [1327].

We are currently focusing on the synthesis and reactivity of iron complexes containing PNP pincer ligands based on the 2,6-diaminopyridine scaffold [28-38]. In these ligands the aromatic pyridine ring and the phosphine moieties are connected via $\mathrm{NH}, \mathrm{N}$-alkyl, or $\mathrm{N}$-aryl linkers. Complexes of the type $[\mathrm{Fe}(\mathrm{PNP}-i \mathrm{Pr})(\mathrm{H})(\mathrm{CO})(\mathrm{L})]^{\mathrm{n}}$ with labile ligands ( $\mathrm{L}=\mathrm{Br}^{-}, \mathrm{CH}_{3} \mathrm{CN}, \mathrm{BH}_{4}{ }^{-}$) and $\mathrm{NH}$ spacers were found to be efficient catalysts for the hydrogenation of both ketones and aldehydes to alcohols under mild conditions (Scheme 1) [35].

Herein we report on the synthesis, characterization, and preliminary catalytic activity of asymmetric chiral iron pincer complexes where $\mathrm{PiPr}_{2}$ and BINEPINE moieties are connected to the pyridine ring of the PNP ligand via $\mathrm{NH}$ spacers. The $i \operatorname{Pr}$ substituents were chosen to prevent the coordination of two PNP ligands as found recently for sterically little demanding PNP systems [34, 36].

\section{Results and discussion}

Treatment of the mono-phosphinated ligand $\mathrm{PN}^{\mathrm{NH} 2}-i \operatorname{Pr}(\mathbf{1})$ with 1 equiv of $\mathrm{BIN}-\mathrm{PCl}(2)$ (in the $R$-form) in the presence of $\mathrm{NEt}_{3}$ afforded the new asymmetric chiral PNP ligand PNP-iPr,BIN (3) in $64 \%$ isolated yield (Scheme 2). The optically pure ligand was isolated as air stable solid and was characterized by elemental analysis, ${ }^{1} \mathrm{H},{ }^{13} \mathrm{C}\left\{{ }^{1} \mathrm{H}\right\}$, and ${ }^{31} \mathrm{P}\left\{{ }^{1} \mathrm{H}\right\}$ NMR spectroscopy.

Treatment of anhydrous $\mathrm{FeCl}_{2}$ with 1 equiv of the PNP ligand $\mathrm{PNP}-i \mathrm{Pr}, \mathrm{BIN}$ (3) in $\mathrm{THF}$ at room temperature afforded the coordinatively unsaturated complex [Fe(PNP$i \mathrm{Pr}, \mathrm{BIN}) \mathrm{Cl}_{2}$ ] (4a) in $79 \%$ isolated yields (Scheme 3 ). The analogous bromide complex [Fe(PNP-iPr,BIN)Br $\left.\mathrm{Br}_{2}\right]$ (4b) was obtained in similar fashion by straightforward complexation of the respective free PNP ligand with anhydrous ferrous dibromide ( $84 \%$ yield). All complexes are thermally robust pale yellow solids that are air sensitive in the solid state and particularly in solution. They display large paramagnetic shifted ${ }^{1} \mathrm{H}$ and ${ }^{13} \mathrm{C}\left\{{ }^{1} \mathrm{H}\right\} \quad \mathrm{NMR}$ solution spectra with broad and featureless signals which, due to the complexity of the PNP ligands, were not assignable and thus not informative. These complexes were characterized by elemental analysis. In addition the molecular structure of $\mathbf{4 d}$ was determined by X-ray crystallography. In this case, the racemic BIN-PCl (2) was used, since all attempts to grow crystals suitable for X-ray diffraction studies failed for the chiral compounds. A structural view of $\mathbf{4 a}$ is depicted in Fig. 1 with selected bond distances and angles given in the caption. The coordination geometry of the iron center is distorted square pyramidal. Bond distances and angles are in good accord with the solid state structures of similar complexes of the type [Fe( $\left.\left.\kappa^{3} P, N, P-\mathrm{PNP}\right) \mathrm{Cl}_{2}\right]$ [29]. Particularly characteristic for all these complexes are the comparatively long $\mathrm{Fe}-\mathrm{N}$ and $\mathrm{Fe}-\mathrm{P}$ bonds which clearly

Scheme 2

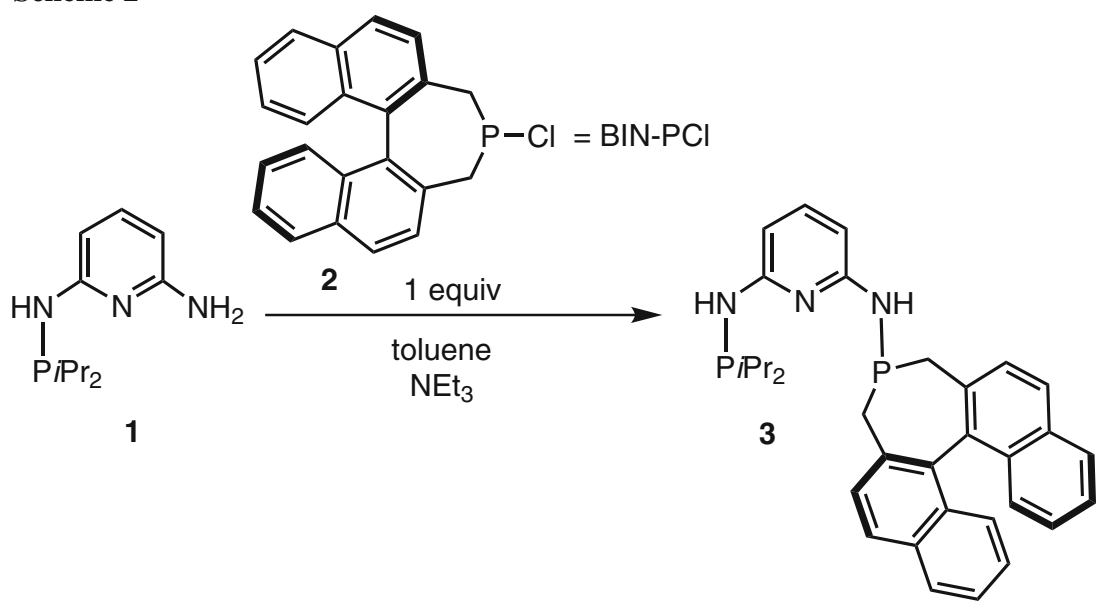


Scheme 3

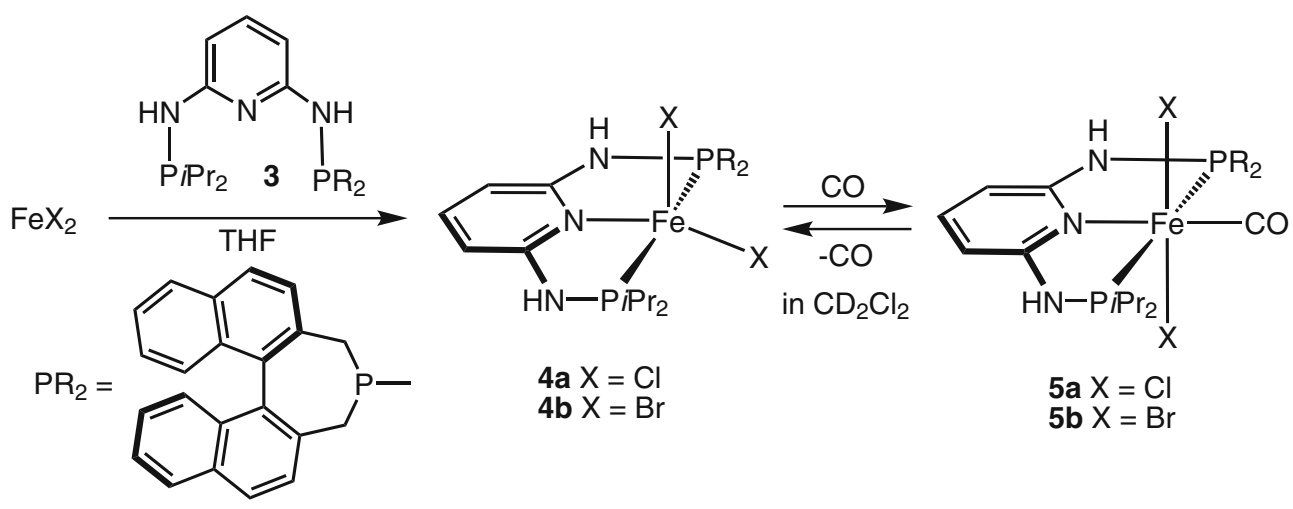

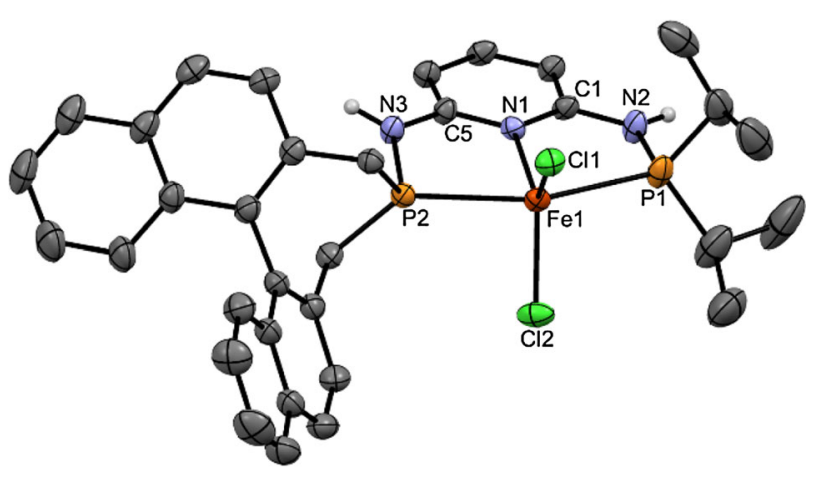

Fig. 1 Structural view of $\left[\mathrm{Fe}(\mathrm{PNP}-i \mathrm{Pr}, \mathrm{BIN}) \mathrm{Cl}_{2}\right]$ (4a) showing $50 \%$ thermal ellipsoids (most $\mathrm{H}$ atoms and solvent molecules omitted for clarity). Selected bond $(\AA)$ and bond angles $\left({ }^{\circ}\right)$ : Fe1-Cl1 2.3057(8), $\mathrm{Fe} 1-\mathrm{Cl} 2$ 2.3366(9), Fe1-P1 2.4853(9), Fe1-P2 2.5150(9), Fe1-N1 2.141(2), Cl1-Fe1-Cl2 130.99(3), Cl1-Fe1-P1 97.69(3), Cl2-Fe1-P2 92.32(3), P1-Fe1-P2 159.16(3), Cl1-Fe1-P1-N2 130.5(1), Cl2-Fe1$\mathrm{P} 1-\mathrm{N} 2$ 97.0(1)

indicate that they are in high-spin state; low-spin Fe-PNP complexes have $\mathrm{Fe}-\mathrm{N}$ and $\mathrm{Fe}-\mathrm{P}$ bonds by ca. $0.2 \AA$ shorter.

In order to obtain iron-based hydrogenation catalysts it appears to be important to have diamagnetic complexes. Accordingly, virtually all iron complexes that are active catalysts feature the strong field $\mathrm{CO}$ and hydride ligands, which seem to maintain a low spin configuration throughout the catalytic cycle. Complexes $\mathbf{4 a}$ and $\mathbf{4 b}$ react readily with the strong $\pi$-acceptor ligand $\mathrm{CO}$ in solution to afford selectively the diamagnetic, octahedral complexes trans-[Fe(PNP-iPr,BIN)(CO)Cl $\left.\mathrm{Cl}_{2}\right](\mathbf{5 a})$ and trans-[Fe(PNP$\left.i \mathrm{Pr}, \mathrm{BIN})(\mathrm{CO}) \mathrm{Br}_{2}\right](\mathbf{5 b})$, respectively, in quantitative yield (Scheme 3). However, due the lability of the CO ligand, these complexes are stable only under a $\mathrm{CO}$ atmosphere and isolation in pure form was not possible. These compounds slowly release $\mathrm{CO}$ thereby reforming the starting materials $4 \mathbf{a}$ and $\mathbf{4 b}$. Accordingly, complexes $5 \mathbf{a}$ and $\mathbf{5 b}$ were fully characterized by ${ }^{1} \mathrm{H},{ }^{13} \mathrm{C}\left\{{ }^{1} \mathrm{H}\right\}$, and ${ }^{31} \mathrm{P}\left\{{ }^{1} \mathrm{H}\right\}$
NMR spectroscopy. In the ${ }^{13} \mathrm{C}\left\{{ }^{1} \mathrm{H}\right\}$ NMR spectrum the CO ligands of $\mathbf{5 a}$ and $\mathbf{5 b}$ exhibit a single low-intensity triplet resonance at $220.7\left(\mathrm{t},{ }^{2} J_{C P}=22.2 \mathrm{~Hz}\right)$ and $223.1 \mathrm{ppm}(\mathrm{t}$, $\left.{ }^{2} J_{C P}=21.9 \mathrm{~Hz}\right)$, respectively. The ${ }^{31} \mathrm{P}\left\{{ }^{1} \mathrm{H}\right\}$ NMR spectra give rise to two doublets centered at 143.6/125.3 and 143.9/ $125.1 \mathrm{ppm}$, respectively, with large $J_{P P}$ coupling constants of 190 and $177 \mathrm{~Hz}$ which are consistent with a trans-P,P configuration.

The preparation of carbonyl hydride complexes was attempted via a one pot procedure by treatment of [Fe(PNP-iPr,BINEP)Br ${ }_{2}$ (4b) with $\mathrm{CO}$ and subsequent reaction with 1.1 equivs of $\mathrm{Na}\left[\mathrm{HBEt}_{3}\right]$ (Scheme 4). Complex $[\mathrm{Fe}(\mathrm{PNP}-i \mathrm{Pr}, \mathrm{BIN})(\mathrm{H})(\mathrm{CO}) \mathrm{Br}](\mathbf{6})$ was obtained as an inseparable mixture of two diastereomers in a ca. 1:1 ratio. Together with 6, a considerable amount of free PNP- $i \mathrm{Pr}$,BIN ligand was detected in the ${ }^{31} \mathrm{P}\left\{{ }^{1} \mathrm{H}\right\}$ NMR spectrum, and the yield was low (ca $50 \%$ ). In good agreement with the experimental findings, also DFT/B3LYP calculations showed that the energy difference between the two isomers (denoted as 6A and 6B) is merely $10.5 \mathrm{~kJ} / \mathrm{mol}$ (Fig. 2). In both isomers, the hydride ligands are located trans to the bromide ligand. Complex 6 turned out to be unstable in solution and slow decomposition took place. Accordingly, complete purification and characterization was unsuccessful. The products were identified by ${ }^{1} \mathrm{H}$ NMR where the hydride signals of the two isomers $6 \mathbf{A}$ and $6 \mathbf{B}$ gave rise to triplet resonances at $-22.15 \quad\left({ }^{2} J_{P H}=61.6 \mathrm{~Hz}\right)$ and $-22.36 \mathrm{ppm}\left({ }^{2} J_{P H}=61.1 \mathrm{~Hz}\right)$, respectively (Fig. 3). The assignments are based on DFT calculations.

Despite these facts, complex $\mathbf{6}$ (as mixture of two diastereomers), prepared in situ, was tested as catalyst for the hydrogenation of some ketones. Since the catalyst could not be isolated in pure form, only preliminary tests were performed with five substrates. These results are depicted in Table 1. The catalyst showed acceptable activity under mild conditions ( 5 bar $\mathrm{H}_{2}$, room temperature) with yields up to $>99 \%$ within $18 \mathrm{~h}$ indicating that in 


\section{Scheme 4}

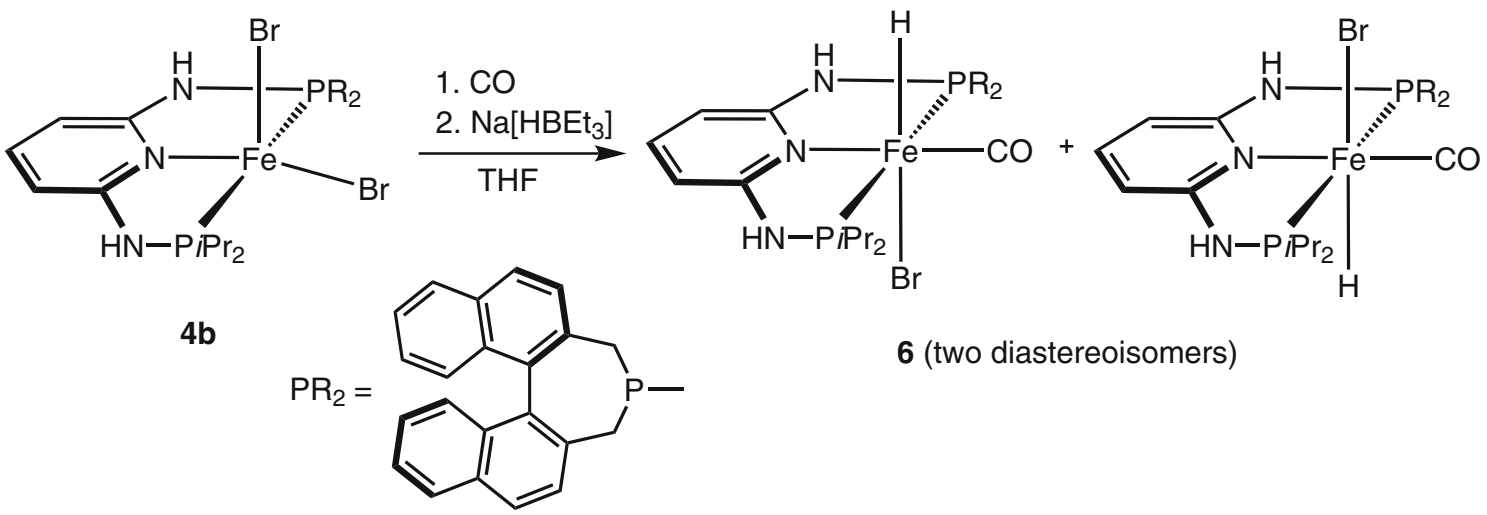

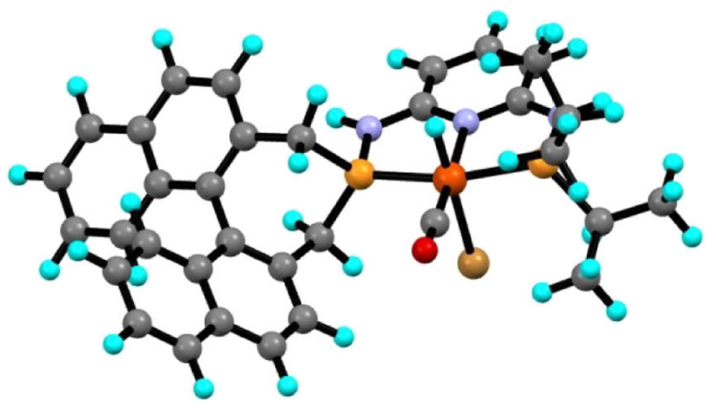

6A $(0.0)$

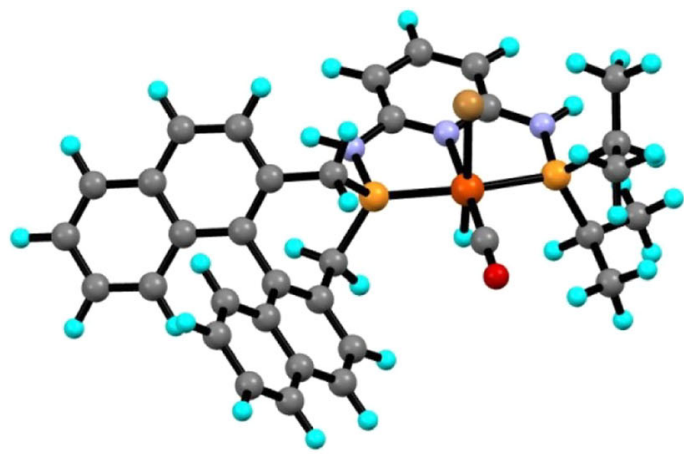

6B $(9.6 \mathrm{~kJ} / \mathrm{mol})$

Fig. 2 DFT/B3LYP optimized structures and free energy difference of the two diasteromers of $[\mathrm{Fe}(\mathrm{PNP}-i \mathrm{Pr}, \mathrm{BIN})(\mathrm{H})(\mathrm{CO}) \mathrm{Br}](\mathbf{6})$

Fig. 3 Hydride region of the ${ }^{1} \mathrm{H}$ NMR spectrum of $\mathbf{6 A}(l e f t)$ and 6B (right) in $\mathrm{CD}_{3} \mathrm{OD}$

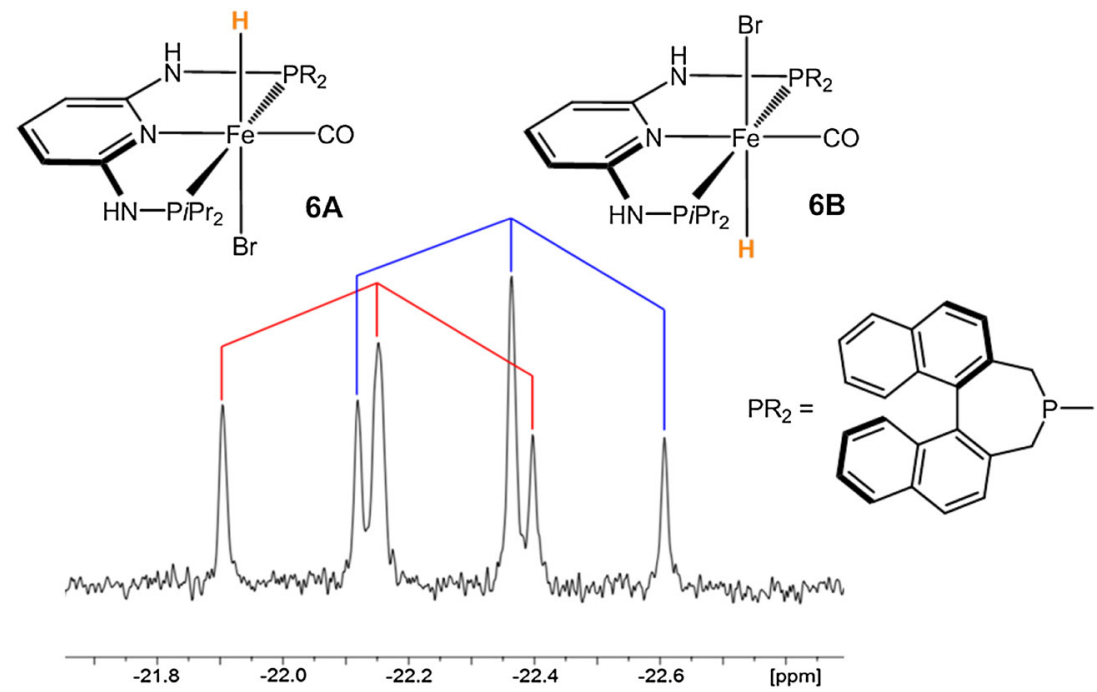

further experiments the catalyst loadings and possibly reaction times can be reduced significantly.

In conclusion, we describe here the synthesis of a new asymmetric chiral PNP ligand based on the 2,6- diaminopyridine scaffold featuring an $R$-BINEPINE moiety. This ligand reacts with anhydrous $\mathrm{FeX}_{2}(\mathrm{X}=\mathrm{Cl}, \mathrm{Br})$ to afford the coordinatively unsaturated paramagnetic complexes $\left[\mathrm{Fe}(\mathrm{PNP}-i \mathrm{Pr}, \mathrm{BIN}) \mathrm{X}_{2}\right]$. Both complexes react 
Table 1 Hydrogenation of ketones catalyzed by [Fe(PNP- $i$ Pr,$\mathrm{BIN})(\mathrm{H})(\mathrm{CO}) \mathrm{Br}](\mathbf{6})$

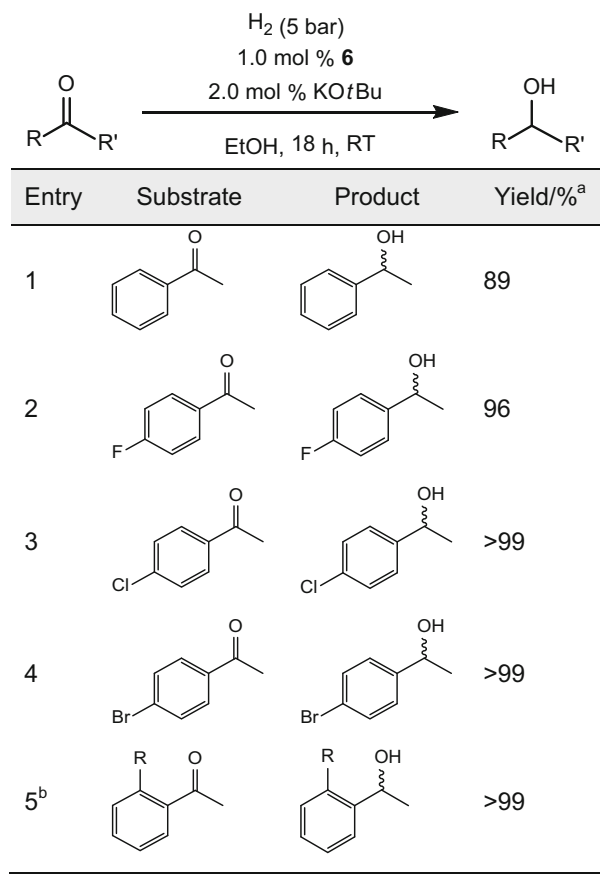

a Yields were determined by ${ }^{1} \mathrm{H}$ NMR

${ }^{\mathrm{b}} R=$ naphtyl

readily with the strong $\pi$-acceptor ligand $\mathrm{CO}$ in solution to afford selectively and quantitatively trans-[Fe(PNP$\left.i \mathrm{Pr}, \mathrm{BIN})(\mathrm{CO}) \mathrm{X}_{2}\right]$. Due the lability of the $\mathrm{CO}$ ligand, these complexes are only stable under a $\mathrm{CO}$ atmosphere. The preparation of the carbonyl hydride complex [Fe(PNP$i \mathrm{Pr}, \mathrm{BIN})(\mathrm{H})(\mathrm{CO}) \mathrm{Br}$ ] was achieved albeit in low yields via a one pot synthesis. This complex was obtained as an inseparable mixture of two diastereomers and was successfully tested as catalyst for the hydrogenation of ketones.

\section{Experimental}

All manipulations were performed under an inert atmosphere of argon by using Schlenk techniques or in an MBraun inertgas glovebox. The solvents were purified according to standard procedures [39]. The deuterated solvents were purchased from Aldrich and dried over $4 \AA$ molecular sieves. The ligands $N^{2}$-(diisopropylphosphanyl)pyridine-2,6-diamine $\left(\mathrm{PN}^{\mathrm{NH} 2}-i \mathrm{Pr}\right)(\mathbf{1})$ and $(1 R)-4$-chloro-4,5-dihydro- $3 H$ dinaphto[2,1-c: $\left.1^{\prime}, 2^{\prime}-e\right]$ phosphepine (BIN-PCl) (2) were prepared according to the literature $[37,40] .{ }^{1} \mathrm{H},{ }^{13} \mathrm{C}\left\{{ }^{1} \mathrm{H}\right\}$, and ${ }^{31} \mathrm{P}\left\{{ }^{1} \mathrm{H}\right\}$ NMR spectra were recorded on Bruker AVANCE-250, AVANCE-300 DPX, and AVANCE-400 spectrometers. ${ }^{1} \mathrm{H}$ and ${ }^{13} \mathrm{C}\left\{{ }^{1} \mathrm{H}\right\}$ NMR spectra were referenced internally to residual protio-solvent, and solvent resonances, respectively, and are reported relative to tetramethylsilane $(\delta=0 \mathrm{ppm}) .{ }^{31} \mathrm{P}\left\{{ }^{1} \mathrm{H}\right\}$ NMR spectra were referenced externally to $\mathrm{H}_{3} \mathrm{PO}_{4}(85 \%)(\delta=0 \mathrm{ppm})$.

(1R)- $N^{2}-(3,5-D i h y d r o-4 H$-dinaphtho[2,1-c:1',2'-e]phosphepin-4-yl)- $N^{6}$-(diisopropylphosphanyl)pyridine-2,6-diamine (PNP-iPr,BIN) $\left(3, \mathrm{C}_{33} \mathrm{H}_{35} \mathrm{~N}_{3} \mathrm{P}_{2}\right)$

1 (1.00 eq, $6.55 \mathrm{mmol}, 1.47 \mathrm{~g}$ ) was dissolved in $100 \mathrm{~cm}^{3}$ toluene and $1.4 \mathrm{~cm}^{3} \mathrm{Et}_{3} \mathrm{~N}$ (1.50 eq, $9.83 \mathrm{mmol}$ ) was added. After cooling to $0{ }^{\circ} \mathrm{C}, 2.50 \mathrm{~g} 2(1.10 \mathrm{eq}, 7.21 \mathrm{mmol})$ in $50 \mathrm{~cm}^{3}$ toluene was added and the reaction was stirred at $80{ }^{\circ} \mathrm{C}$ for $12 \mathrm{~h}$. The suspension was filtered over a small pad of Celite ${ }^{\circledR}$ and the solvent was removed under reduced pressure. The crude product was purified via flash chromatography using silica gel (conditioned with 5 vol $\% \mathrm{NEt}_{3}$ ) and $1: 1 \mathrm{MC} /$ EE as eluent. Yield: $2.25 \mathrm{~g}(64 \%) .{ }^{1} \mathrm{H} \mathrm{NMR}\left(\mathrm{CDCl}_{3}, 20{ }^{\circ} \mathrm{C}\right)$ : $\delta=7.89-7.79$ (m, 4H, naph), $7.47\left(\mathrm{~d},{ }^{3} J_{H H}=8.3 \mathrm{~Hz}, 1 \mathrm{H}\right.$, naph), 7.38-7.32 (m, 2H, naph), $7.31\left(\mathrm{~d},{ }^{3} J_{H H}=8.3 \mathrm{~Hz}, 1 \mathrm{H}\right.$, naph), $7.23\left(\mathrm{~m}, 5 \mathrm{H}, \mathrm{py}^{4}\right.$, naph), $6.43\left(\mathrm{dd},{ }^{3} J_{H H}=8.0 \mathrm{~Hz}\right.$, $\left.{ }^{4} J_{P H}=1.9 \mathrm{~Hz}, 1 \mathrm{H}, \mathrm{py}^{5}\right), 6.22\left(\mathrm{~d},{ }^{3} J_{H H}=8.4 \mathrm{~Hz}, 1 \mathrm{H}, \mathrm{py}^{3}\right)$, $4.31\left(\mathrm{~d}, \quad{ }^{2} J_{P H}=10.9 \mathrm{~Hz}, \quad 1 \mathrm{H}, \quad \mathrm{N} H^{\mathrm{BIN}}\right), \quad 4.08 \quad(\mathrm{~d}$, $\left.{ }^{2} J_{P H}=10.7 \mathrm{~Hz}, 1 \mathrm{H}, \mathrm{N} H^{i \mathrm{Pr}}\right), 3.03\left(\mathrm{dd},{ }^{2} J_{H H}=17.0 \mathrm{~Hz}\right.$, $\left.{ }^{2} J_{P H}=11.9 \mathrm{~Hz}, 1 \mathrm{H}, \mathrm{CH}_{2}\right), 2.70\left(\mathrm{dd},{ }^{2} J_{H H}=14.3 \mathrm{~Hz}\right.$, $\left.{ }^{2} J_{P H}=2.8 \mathrm{~Hz}, 1 \mathrm{H}, \mathrm{CH}_{2}\right), 2.50\left(\mathrm{dd},{ }^{2} J_{H H}=18.2 \mathrm{~Hz}\right.$, $\left.{ }^{2} J_{P H}=14.3 \mathrm{~Hz}, 1 \mathrm{H}, \mathrm{CH}_{2}\right), 2.29\left(\mathrm{~d},{ }^{2} J_{H H}=11.9 \mathrm{~Hz}, 1 \mathrm{H}\right.$, $\left.\mathrm{CH}_{2}\right), 1.68\left(\mathrm{~m}, 2 \mathrm{H}, \mathrm{CH}\left(\mathrm{CH}_{3}\right)_{2}\right), 1.04-0.95(\mathrm{~m}, 12 \mathrm{H}$, $\left.\mathrm{CH}\left(\mathrm{CH}_{3}\right)_{2}\right) \quad \mathrm{ppm} ; \quad{ }^{13} \mathrm{C}\left\{{ }^{1} \mathrm{H}\right\} \quad \mathrm{NMR} \quad\left(\mathrm{CDCl}_{3}, \quad 20{ }^{\circ} \mathrm{C}\right)$ : $\delta=159.92 \quad\left(\mathrm{~d}, \quad{ }^{2} J_{C P}=20.0 \mathrm{~Hz}, \quad \mathrm{py}^{6}\right), \quad 157.01 \quad(\mathrm{~d}$, $\left.{ }^{2} J_{C P}=17.4, \mathrm{py}^{2}\right), 139.25\left(\mathrm{~s}, \mathrm{py}^{4}\right), 133.79\left(\mathrm{~d}, J_{C P}=4.1 \mathrm{~Hz}\right.$, naph), 133.43 (s, naph), 133.08 (s, naph), 132.82 (d, $J_{C P}=1.5 \mathrm{~Hz}$, naph), 132.72 (s, naph), 132.38 (s, naph), 132.28 (d, $J_{C P}=1.8 \mathrm{~Hz}$, naph), 132.15 (s, naph), 128.24 (s, naph), 127.58 (s, naph), $127.23\left(\mathrm{~d}, J_{C P}=2.1 \mathrm{~Hz}\right.$, naph), $126.69\left(\mathrm{~d}, J_{C P}=10.8 \mathrm{~Hz}\right.$, naph), $126.06\left(\mathrm{~d}, J_{C P}=11.5 \mathrm{~Hz}\right.$, naph), $125.09\left(\mathrm{~d}, \quad J_{C P}=11.9 \mathrm{~Hz}, \quad\right.$ naph $), 98.90 \quad(\mathrm{~d}$, $\left.{ }^{3} J_{C P}=18.4 \mathrm{~Hz}, \mathrm{py}^{5}\right), 98.73\left(\mathrm{~d},{ }^{3} J_{C P}=15.7, \mathrm{py}^{3}\right), 36.04(\mathrm{~d}$, $\left.{ }^{1} J_{C P}=15.0 \mathrm{~Hz}, C \mathrm{H}_{2}\right), 34.90\left(\mathrm{~d},{ }^{1} J_{C P}=24.2 \mathrm{~Hz}, C \mathrm{H}_{2}\right)$, $26.43\left(\mathrm{~d}, \quad{ }^{1} J_{C P}=11.5 \mathrm{~Hz}, \quad C \mathrm{H}\left(\mathrm{CH}_{3}\right)_{2}\right), \quad 16.34 \quad(\mathrm{~d}$, $\left.{ }^{1} J_{C P}=11.0 \mathrm{~Hz}, C \mathrm{H}\left(\mathrm{CH}_{3}\right)_{2}\right), 18.72\left(\mathrm{~d},{ }^{2} J_{C P}=19.8 \mathrm{~Hz}\right.$, $\left.\mathrm{CH}\left(\mathrm{CH}_{3}\right)_{2}\right), 17.22\left(\mathrm{~d},{ }^{2} J_{\mathrm{CP}}=10.6 \mathrm{~Hz}, \mathrm{CH}\left(\mathrm{CH}_{3}\right)_{2}\right), 17.14$ $\left(\mathrm{d},{ }^{2} J_{C P}=10.4 \mathrm{~Hz}, \mathrm{CH}\left(\mathrm{CH}_{3}\right)_{2}\right) \mathrm{ppm} ;{ }^{31} \mathrm{P}\left\{{ }^{1} \mathrm{H}\right\} \mathrm{NMR}\left(\mathrm{CDCl}_{3}\right.$, $\left.20{ }^{\circ} \mathrm{C}\right): \delta=48.6(\mathrm{~s}, i \operatorname{Pr}), 48.1$ (s, BIN) ppm.

[(Dichloro) $(1 R)-N^{2}-(3,5-$ dihydro-4H-dinaphtho[2,1$c: 1^{\prime}, 2^{\prime}-$ e]phosphepin-4-yl)-N6-(diisopropylphosphanyl)pyridine-2,6-diamine)iron(II)] ([Fe(PNP-iPr, BIN)Cl $l_{2}$ ) (4a, $\mathrm{C}_{33} \mathrm{H}_{35} \mathrm{Cl}_{2} \mathrm{FeN}_{3} \mathrm{P}_{2}$ )

A suspension of $71 \mathrm{mg}$ anhydrous $\mathrm{FeCl}_{2}(0.56 \mathrm{mmol})$ and $300 \mathrm{mg} 3(0.56 \mathrm{mmol})$ was stirred in $15 \mathrm{~cm}^{3}$ of THF at 
room temperature for $12 \mathrm{~h}$. The solvent was then removed under vacuum and the remaining solid dissolved in $15 \mathrm{~cm}^{3}$ of $\mathrm{CH}_{2} \mathrm{Cl}_{2}$. Insoluble materials were removed by filtration. The volume of the solution was reduced to $0.5 \mathrm{~cm}^{3}$ and the product was precipitated by addition of $40 \mathrm{~cm}^{3}$ of $n$ pentane. After filtration the yellow product was washed with twice with $15 \mathrm{~cm}^{3}$ of $n$-pentane and dried under vacuum. Yield: $324 \mathrm{mg}(87 \%)$ yellow solid.

[(Dibromo)(1R)- $N^{2}$-(3,5-dihydro-4H-dinaphtho[2,1$c: 1^{\prime}, 2^{\prime}$-e]phosphepin-4-yl)- $N^{6}$-(diisopropylphosphanyl)pyridine-2,6-diamine)iron(II)] ([Fe(PNP-iPr, $\left.\left.B I N) B r_{2}\right]\right)\left(\mathbf{4} \mathbf{b}, \mathrm{C}_{33} \mathrm{H}_{35} \mathrm{Br}_{2} \mathrm{FeN}_{3} \mathrm{P}_{2}\right)$

This complex was prepared analogously to $4 \mathbf{a}$ with $121 \mathrm{mg}$ anhydrous $\mathrm{FeBr}_{2}(0.56 \mathrm{mmol})$ and $300 \mathrm{mg} 3(0.56 \mathrm{mmol})$ as starting materials. Yield: $352 \mathrm{mg}(84 \%)$, yellow solid.

Reaction of $\left[\mathrm{Fe}(\mathrm{PNP}-\mathrm{iPr}, \mathrm{BIN}) \mathrm{Cl}_{2}\right](\mathbf{4 a})$ with $\mathrm{CO}$ in $\mathrm{CD}_{2}$ $\mathrm{Cl}_{2}$. Formation of trans-[(dichloro $)($ carbonyl $)(1 R)-N^{2}-(3,5-$ dihydro-4H-dinaphtho[2,1-c: $\left.\left.1^{\prime}, 2^{\prime}-e\right] p h o s p h e p i n-4-y l\right)-N^{6}$ (diisopropylphosphanyl)pyridine-2,6-diamine)iron(II)] (trans- $\left.\left[\mathrm{Fe}(\mathrm{PNP}-\mathrm{iPr}, \mathrm{BIN})(\mathrm{CO}) \mathrm{Cl}_{2}\right]\right)\left(\mathbf{5 a}, \mathrm{C}_{34} \mathrm{H}_{35} \mathrm{Cl}_{2}\right.$ $\mathrm{FeN}_{3} \mathrm{OP}_{2}$ )

$\mathrm{CO}$ cwas bubbled through a solution of $30 \mathrm{mg} \mathbf{4 a}(45.3 \mu \mathrm{mol})$ in $0.6 \mathrm{~cm}^{3}$ of $\mathrm{CD}_{2} \mathrm{Cl}_{2}$ for $2 \mathrm{~min}$, whereupon the colour changed to dark violet. ${ }^{1} \mathrm{H}$ NMR $\left(\mathrm{CD}_{2} \mathrm{Cl}_{2}, 20{ }^{\circ} \mathrm{C}\right): \delta=8.13-8.00(\mathrm{~m}$, $4 \mathrm{H}$, naph), $7.79\left(\mathrm{~d},{ }^{3} J_{H H}=7.9 \mathrm{~Hz}, 1 \mathrm{H}\right.$, naph $), 7.66(\mathrm{~d}$, ${ }^{3} J_{H H}=7.5 \mathrm{~Hz}, 1 \mathrm{H}$, naph), $7.41\left(\mathrm{~d},{ }^{3} J_{H H}=8.4 \mathrm{~Hz}, 1 \mathrm{H}\right.$, naph), 7.34-7.15 (m, 6H, naph, py $\left.{ }^{4}\right), 6.62\left(\mathrm{bs}, 1 \mathrm{H}, \mathrm{py}^{5}\right), 6.41(\mathrm{bs}, 1 \mathrm{H}$, $\left.\mathrm{N} H^{\mathrm{iPr}}\right), 6.03\left(\mathrm{bs}, 1 \mathrm{H}, \mathrm{py}^{3}\right), 5.56\left(\mathrm{bs}, 1 \mathrm{H}, \mathrm{N} H^{\mathrm{BIN}}\right), 4.35(\mathrm{dd}$, $\left.{ }^{2} J_{H H}=12.3 \mathrm{~Hz}, \quad{ }^{2} J_{P H}=4.1 \mathrm{~Hz}, 1 \mathrm{H}, \quad \mathrm{CH}_{2}\right), 3.76(\mathrm{dd}$, $\left.{ }^{2} J_{H H}=15.1 \mathrm{~Hz}, \quad{ }^{2} J_{P H}=9.1 \mathrm{~Hz}, \quad 1 \mathrm{H}, \quad \mathrm{CH}_{2}\right), \quad 3.20 \quad(\mathrm{~d}$, $\left.{ }^{2} J_{H H}=15.0 \mathrm{~Hz}, 1 \mathrm{H}, \mathrm{CH}_{2}\right), 2.99\left(\mathrm{~m}, 2 \mathrm{H}, \mathrm{CH}\left(\mathrm{CH}_{3}\right)_{2}\right), 2.71$ $\left(\mathrm{dd},{ }^{2} J_{H H}=17.0 \mathrm{~Hz},{ }^{2} J_{P H}=13.0 \mathrm{~Hz}, 1 \mathrm{H}, \mathrm{CH}_{2}\right), 1.62-1.37$ (m, 12H, $\left.\mathrm{CH}\left(\mathrm{CH}_{3}\right)_{2}\right) \mathrm{ppm} ;{ }^{13} \mathrm{C}\left\{{ }^{1} \mathrm{H}\right\} \mathrm{NMR}\left(\mathrm{CD}_{2} \mathrm{Cl}_{2}, 20{ }^{\circ} \mathrm{C}\right)$ : $\delta=220.73 \quad\left(\mathrm{t}, \quad{ }^{2} J_{C P}=22.2 \mathrm{~Hz}, \quad C \mathrm{C}\right), \quad 161.90 \quad(\mathrm{dd}$, $\left.{ }^{2} J_{C P}=13.2 \mathrm{~Hz}, \quad{ }^{3} J_{C P}=5.6 \mathrm{~Hz}, \quad \mathrm{py}^{6}\right), \quad 160.63 \quad(\mathrm{dd}$, $\left.{ }^{2} J_{C P}=13.6 \mathrm{~Hz},{ }^{3} J_{C P}=5.1 \mathrm{~Hz}, \mathrm{py}^{2}\right), 140.16 \quad\left(\mathrm{~s}, \mathrm{py}^{4}\right)$, $134.67\left(\mathrm{~d}, J_{C P}=1.8 \mathrm{~Hz}\right.$, naph), $134.16\left(\mathrm{~d}, J_{C P}=4.9 \mathrm{~Hz}\right.$, naph), 133.21 (d, $J_{C P}=2.6 \mathrm{~Hz}$, naph), 132.91 (d, $J_{C P}=1.6$ $\mathrm{Hz}$, naph), 132.82 (s, naph), 132.49 (s, naph), 132.24 (d, $J_{C P}=2.5 \mathrm{~Hz}$, naph), $131.56\left(\mathrm{~d}, J_{C P}=3.0 \mathrm{~Hz}\right.$, naph), 129.03 (s, naph), $128.81\left(\mathrm{~d}, J_{C P}=2.1 \mathrm{~Hz}\right.$, naph), 128.45 (d, $J_{C P}=3.6 \mathrm{~Hz}$, naph), $128.37 \quad\left(\mathrm{~d}, J_{C P}=8.0 \mathrm{~Hz}\right.$, naph), 127.53 (s, naph), 126.96 (s, naph), 126.79 (s, naph), 126.40 (s, naph), 126.08 (s, naph), 125.65 (s, naph), 125.51 (s, naph), $99.88\left(\mathrm{~d},{ }^{3} J_{C P}=7.7 \mathrm{~Hz}, \mathrm{py}{ }^{5}\right), 99.52\left(\mathrm{~d},{ }^{3} J_{C P}=7.2 \mathrm{~Hz}, \mathrm{py}^{3}\right)$, $33.05\left(\mathrm{~d},{ }^{1} J_{C P}=21.9 \mathrm{~Hz}, C \mathrm{H}_{2}\right), 29.10\left(\mathrm{~d},{ }^{1} J_{C P}=26.3 \mathrm{~Hz}\right.$, $\left.\mathrm{CH}_{2}\right), 26.23\left(\mathrm{~d},{ }^{1} J_{C P}=21.9 \mathrm{~Hz}, C \mathrm{CH}\left(\mathrm{CH}_{3}\right)_{2}\right), 25.95(\mathrm{~d}$, $\left.{ }^{1} J_{C P}=22.2 \mathrm{~Hz}, \quad C \mathrm{H}\left(\mathrm{CH}_{3}\right)_{2}\right), 18.88 \quad\left(\mathrm{~d},{ }^{2} J_{C P}=4.1 \mathrm{~Hz}\right.$, $\left.\mathrm{CH}\left(\mathrm{CH}_{3}\right)_{2}\right), 18.86\left(\mathrm{~d},{ }^{2} J_{C P}=4.0 \mathrm{~Hz}, \mathrm{CH}\left(\mathrm{CH}_{3}\right)_{2}\right), 17.95(\mathrm{~d}$, $\left.{ }^{2} J_{C P}=3.5 \mathrm{~Hz}, \mathrm{CH}\left(\mathrm{CH}_{3}\right)_{2}\right)$ ppm; ${ }^{31} \mathrm{P}\left\{{ }^{1} \mathrm{H}\right\}$ NMR $\left(\mathrm{CD}_{2} \mathrm{Cl}_{2}\right.$, $\left.20{ }^{\circ} \mathrm{C}\right): \delta=143.6\left(\mathrm{~d},{ }^{2} J_{P P}=189.7 \mathrm{~Hz}, \mathrm{BIN}\right), 125.3(\mathrm{~d}$, $\left.{ }^{2} J_{P P}=189.7 \mathrm{~Hz}, i \operatorname{Pr}\right) \mathrm{ppm}$.
Reaction of $\left[\mathrm{Fe}(\mathrm{PNP}-\mathrm{iPr}, \mathrm{BIN}) \mathrm{Br}_{2}\right](\mathbf{4 b})$ with $\mathrm{CO}$ in $\mathrm{CD}_{2}$ $\mathrm{Cl}_{2}$. Formation of trans-[(dibromo $)($ carbonyl $)(1 \mathrm{R})-\mathrm{N}^{2}-(3,5-$ dihydro-4H-dinaphtho[2,1-c:1', $2^{\prime}$-e $]$ phosphepin-4-yl)- $N^{6}$ (diisopropylphosphanyl)pyridine-2,6-diamine)iron(II)] (trans-[Fe $\left.\left.(P N P-i P r, B I N)(C O) B_{2}\right]\right)\left(\mathbf{5 b}, \mathrm{C}_{34} \mathrm{H}_{35} \mathrm{Br}_{2}\right.$ $\mathrm{FeN}_{3} \mathrm{OP}_{2}$ )

CO was bubbled through a solution of $30 \mathrm{mg} \mathbf{4 b}$ $(39.9 \mu \mathrm{mol})$ in $0.6 \mathrm{~cm}^{3}$ of $\mathrm{CD}_{2} \mathrm{Cl}_{2}$ for $2 \mathrm{~min}$, whereupon the colour changed to dark violet. ${ }^{1} \mathrm{H}$ NMR $\left(\mathrm{CD}_{2} \mathrm{Cl}_{2}\right.$, $\left.20{ }^{\circ} \mathrm{C}\right): \quad \delta=8.01-7.90 \quad(\mathrm{~m}, \quad 4 \mathrm{H}, \quad$ naph $), \quad 7.80 \quad(\mathrm{~d}$, ${ }^{3} J_{H H}=7.9 \mathrm{~Hz}, 1 \mathrm{H}$, naph $), 7.54\left(\mathrm{~d},{ }^{3} J_{H H}=7.5 \mathrm{~Hz}, 1 \mathrm{H}\right.$, naph), 7.41-7.36 (m, 3H, naph), $7.30\left(\mathrm{~d},{ }^{3} J_{H H}=8.4 \mathrm{~Hz}\right.$, $1 \mathrm{H}$, naph), 7.25-7.03 (m, 3H, naph, py $\left.{ }^{4}\right), 6.50$ (bs, $1 \mathrm{H}$, $\left.\mathrm{py}^{5}\right), 6.32\left(\mathrm{~d},{ }^{2} J_{P H}=5.9 \mathrm{~Hz}, 1 \mathrm{H}, \mathrm{N} H^{\mathrm{iPr}}\right), 5.83(\mathrm{bs}, 1 \mathrm{H}$, $\left.\mathrm{py}^{3}\right), 5.50\left(\mathrm{~d},{ }^{2} J_{P H}=6.0 \mathrm{~Hz}, 1 \mathrm{H}, \quad \mathrm{N} H^{\mathrm{BIN}}\right), 4.67(\mathrm{dd}$, $\left.{ }^{2} J_{H H}=12.5 \mathrm{~Hz},{ }^{2} J_{P H}=3.9 \mathrm{~Hz}, 1 \mathrm{H}, \mathrm{CH}_{2}\right), 3.94(\mathrm{dd}$, $\left.{ }^{2} J_{H H}=15.1 \mathrm{~Hz},{ }^{2} J_{P H}=8.8 \mathrm{~Hz}, 1 \mathrm{H}, \mathrm{CH}_{2}\right), 3.10(\mathrm{~m}, 3 \mathrm{H}$, $\left.\mathrm{CH}_{2}, \quad \mathrm{CH}\left(\mathrm{CH}_{3}\right)_{2}\right), \quad 2.73 \quad\left(\mathrm{dd}, \quad{ }^{2} J_{H H}=17.3 \mathrm{~Hz}\right.$, $\left.{ }^{2} J_{P H}=13.3 \mathrm{~Hz}, 1 \mathrm{H}, \mathrm{CH}_{2}\right), 1.49\left(\mathrm{dd},{ }^{3} J_{H H}=5.8 \mathrm{~Hz}\right.$, $\left.{ }^{2} J_{P H}=12.1 \mathrm{~Hz}, \quad 3 \mathrm{H}, \quad \mathrm{CH}\left(\mathrm{CH}_{3}\right)_{2}\right), \quad 1.45 \quad(\mathrm{dd}$, $\left.{ }^{3} J_{H H}=6.8 \mathrm{~Hz},{ }^{2} J_{P H}=15.3 \mathrm{~Hz}, 3 \mathrm{H}, \quad \mathrm{CH}\left(\mathrm{CH}_{3}\right)_{2}\right), 1.41$ $\left(\mathrm{dd},{ }^{3} J_{H H}=7.2 \mathrm{~Hz},{ }^{2} J_{P H}=16.9 \mathrm{~Hz}, 3 \mathrm{H}, \mathrm{CH}\left(\mathrm{CH}_{3}\right)_{2}\right)$, $1.32\left(\mathrm{dd},{ }^{3} J_{H H}=7.2 \mathrm{~Hz},{ }^{2} J_{P H}=16.6 \mathrm{~Hz}, 3 \mathrm{H}, \mathrm{CH}\left(\mathrm{CH}_{3}\right)_{2}\right)$ ppm; ${ }^{13} \mathrm{C}\left\{{ }^{1} \mathrm{H}\right\}$ NMR $\left(\mathrm{CD}_{2} \mathrm{Cl}_{2}, 20{ }^{\circ} \mathrm{C}\right): \delta=223.05(\mathrm{t}$, $\left.{ }^{2} J_{C P}=21.9 \mathrm{~Hz}, \quad C \mathrm{O}\right), \quad 161.97 \quad\left(\mathrm{dd}, \quad{ }^{2} J_{C P}=12.6 \mathrm{~Hz}\right.$, ${ }^{3} J_{C P}=5.3 \mathrm{~Hz}, \quad$ py $\left.{ }^{6}\right), \quad 160.60 \quad\left(\mathrm{dd}, \quad{ }^{2} J_{C P}=13.3 \mathrm{~Hz}\right.$, $\left.{ }^{3} J_{C P}=5.1 \mathrm{~Hz}, \mathrm{py}^{2}\right), 140.11\left(\mathrm{~s}, \mathrm{py}^{4}\right), 134.65$ (s, naph), $134.12\left(\mathrm{~d}, J_{C P}=5.0 \mathrm{~Hz}\right.$, naph), $133.67\left(\mathrm{~d}, J_{C P}=10.9 \mathrm{~Hz}\right.$, naph), 133.18 (d, $J_{C P}=2.6 \mathrm{~Hz}$, naph), 132.92 (d, $J_{C P}=1.3 \mathrm{~Hz}$, naph), 132.44 (s, naph), 132.18 (d, $J_{C P}=2.4 \mathrm{~Hz}$, naph), $131.61\left(\mathrm{~d}, J_{C P}=2.9 \mathrm{~Hz}\right.$, naph), 129.08 (s, naph), 128.80 (d, $J_{C P}=2.2 \mathrm{~Hz}$, naph), 128.39 (d, $J_{C P}=6.2 \mathrm{~Hz}$, naph), $128.22\left(\mathrm{~d}, J_{C P}=3.5 \mathrm{~Hz}\right.$, naph), $127.63\left(\mathrm{~d}, J_{C P}=1.6 \mathrm{~Hz}\right.$, naph), $126.92\left(\mathrm{~d}, J_{C P}=13.8 \mathrm{~Hz}\right.$, naph), 126.41 (s, naph), 126.10 (s, naph), 125.61 (d, $J_{C P}=13.5 \mathrm{~Hz}$, naph), $100.17\left(\mathrm{~d},{ }^{3} J_{C P}=7.0 \mathrm{~Hz}, \mathrm{py}^{5}\right)$, $99.67\left(\mathrm{~d},{ }^{3} J_{C P}=7.2 \mathrm{~Hz}, \mathrm{py}^{3}\right), 36.23\left(\mathrm{~d},{ }^{1} J_{C P}=23.3 \mathrm{~Hz}\right.$, $\left.\mathrm{CH}_{2}\right), \quad 31.39\left(\mathrm{~d},{ }^{1} J_{\mathrm{CP}}=27.9 \mathrm{~Hz}, \quad \mathrm{CH}_{2}\right), \quad 28.48 \quad(\mathrm{~d}$, $\left.{ }^{1} J_{C P}=22.6 \mathrm{~Hz}, C \mathrm{H}\left(\mathrm{CH}_{3}\right)_{2}\right), 28.01\left(\mathrm{~d},{ }^{1} J_{C P}=23.3 \mathrm{~Hz}\right.$, $\left.\mathrm{CH}\left(\mathrm{CH}_{3}\right)_{2}\right), 18.97\left(\mathrm{~d},{ }^{2} J_{C P}=4.5 \mathrm{~Hz}, \mathrm{CH}\left(\mathrm{CH}_{3}\right)_{2}\right), 18.52$ $\left(\mathrm{m}, \mathrm{CH}\left(\mathrm{CH}_{3}\right)_{2}\right)$ ppm; ${ }^{31} \mathrm{P}\left\{{ }^{1} \mathrm{H}\right\}$ NMR $\left(\mathrm{CD}_{2} \mathrm{Cl}_{2}, 20{ }^{\circ} \mathrm{C}\right)$ : $\delta=143.9 \quad\left(\mathrm{~d}, \quad{ }^{2} J_{P P}=176.6 \mathrm{~Hz}, \quad \mathrm{BIN}\right), \quad 125.1 \quad(\mathrm{~d}$, $\left.{ }^{2} J_{P P}=176.5 \mathrm{~Hz}, i \operatorname{Pr}\right) \mathrm{ppm}$.

Reaction of $\left[\mathrm{Fe}(\mathrm{PNP}-\mathrm{iPr}, \mathrm{BIN}) \mathrm{Br}_{2}\right]$ (4b) with $\mathrm{CO}$ and Na[HBEt $\left.t_{3}\right]$. Formation of [(bromo)(hydrido)(carbonyl)(1R)- $N^{2}-\left(3,5-d i h y d r o-4 H\right.$-dinaphtho[2,1-c: $1^{\prime}, 2^{\prime}$ e]phosphepin-4-yl)- $N^{6}$-(diisopropylphosphanyl)pyridine2,6-diamine)iron(II)] ([Fe(PNP-iPr,BIN)(H)(CO)Br]) (6, $\mathrm{C}_{34} \mathrm{H}_{36} \mathrm{BrFeN}_{3} \mathrm{OP}_{2}$ )

A solution of $300 \mathrm{mg} \mathbf{4 b}(0.40 \mathrm{mmol})$ in $15 \mathrm{~cm}^{3}$ THF was purged with $\mathrm{CO}$ for $3 \mathrm{~min}$, whereupon the colour changed to deep blue. The reaction mixture was then cooled to $0{ }^{\circ} \mathrm{C}$ 
and $0.44 \mathrm{~cm}^{3} \mathrm{Na}\left[\mathrm{HBEt}_{3}\right](0.44 \mathrm{mmol})$ was added slowly via syringe. The solvent was then removed under reduced pressure. The residue was redissolved in $15 \mathrm{~cm}^{3}$ of $\mathrm{CH}_{2} \mathrm{Cl}_{2}$, filtered and the volume of the solution was reduced to ca $0.5 \mathrm{~cm}^{3}$. The product was precipitated upon addition of $40 \mathrm{~cm}^{3}$ of $n$-pentane, collected on a glass frit, washed with $10 \mathrm{~cm}^{3} n$-pentane and dried under vacuum for $2 \mathrm{~h}$. The product could not be isolated in pure form and was used for catalytic reactions as obtained.

\section{X-ray structure determination}

X-ray diffraction data of $\mathbf{4 a} \cdot x \mathrm{THF} \cdot(2-x) \mathrm{Et}_{2} \mathrm{O}$ (CCDC number 1445976) were collected at $T=100 \mathrm{~K}$ in a dry stream of nitrogen on a Bruker Kappa APEX II diffractometer system using graphite-monochromatized $\mathrm{MoK} \alpha$ radiation $(\lambda=0.71073 \AA)$ and fine sliced $\varphi$ - and $\omega$-scans. Data were reduced to intensity values with SAINT and an absorption correction was applied with the multi-scan approach implemented in SADABS [41]. The structures were solved by charge flipping using SUPERFLIP [42] and refined against $F$ with JANA2006 [43]. The electron density in distinct voids of the structure could be attributed to two solvent positions. On one was located an $\mathrm{Et}_{2} \mathrm{O}$ molecule and the other was substitutionally disordered by $\mathrm{Et}_{2} \mathrm{O}$ and THF molecules. Since no refinement with reasonable ADPs of the solvent molecules could be obtained, contributions of the solvent molecules to the diffraction data were removed using the SQUEEZE procedure of PLATON [44]. The non-hydrogen atoms were refined anisotropically. The $\mathrm{H}$ atoms connected to $\mathrm{C}$ atoms were placed in calculated positions and thereafter refined as riding on the parent atoms. $\mathrm{H}$ atoms connected to $\mathrm{N}$ located in difference Fourier maps. Since the point group $4 / m$ of the crystal is a merohedry twinning via twofold rotation about [110] was included in the model. Such models did not result in improved residuals and twinning was ultimately dropped from the refinement. Molecular graphics were generated with the program MERCURY [45]. Crystal data are given in Table S1.

\section{Computational details}

Calculations were performed using the GaUSSIAN 09 software package [46], and the B3LYP functional [47-49] without symmetry constraints. This functional was shown to perform well in mechanistic studies of spin forbidden reactions in closely related $\mathrm{Fe}$ system. The optimized geometries were obtained with the Stuttgart/Dresden ECP (SDD) basis set [50-52] to describe the electrons of the iron atom. For all other atoms a standard $6-31 \mathrm{G}^{* *}$ basis set was employed [53-58]. Frequency calculations were performed to confirm the nature of the stationary points yielding no imaginary frequency for the minima.

Acknowledgments Open access funding provided by Austrian Science Fund (FWF). Financial support by the Austrian Science Fund (FWF) is gratefully acknowledged (Project No. P24583-N28). The X-ray center of the Vienna University of Technology is acknowledged for financial support and for providing access to the singlecrystal diffractometer.

Open Access This article is distributed under the terms of the Creative Commons Attribution 4.0 International License (http:// creativecommons.org/licenses/by/4.0/), which permits unrestricted use, distribution, and reproduction in any medium, provided you give appropriate credit to the original author(s) and the source, provide a link to the Creative Commons license, and indicate if changes were made.

\section{References}

1. Noyori R, Ohkuma T (2001) Angew Chem Int Ed 40:40

2. Noyori R (2013) Angew Chem Int Ed 52:79

3. de Vries JG, Elsevier CJ (eds) (2007) Handbook of homogeneous hydrogenation. Wiley, Weinheim

4. Johnson NB, Lennon IC, Moran PH, Ramsden JA (2007) Acc Chem Res 40:1291

5. Dub PA, Ikariya T (2012) ACS Catal 2:1718

6. Morris RH (2009) Chem Soc Rev 38:2282

7. Enthaler S, Junge K, Beller M (2008) Angew Chem Int Ed 47:3317

8. Gaillard S, Renaud J-L (2008) ChemSusChem 1:505

9. Bauer G, Kirchner KA (2011) Angew Chem Int Ed 50:5798

10. Sues PE, Demmans Z, Morris RH (2014) Dalton Trans 43:7650

11. Bullock RM (2013) Science 342:1054

12. Bauer I, Knölker H-J (2015) Chem Rev 115:3170

13. Casey CP, Guan H (2007) J Am Chem Soc 129:5816

14. Casey CP, Guan H (2009) J Am Chem Soc 131:2499

15. Langer R, Leitus G, Ben-David Y, Milstein D (2011) Angew Chem Int Ed 50:2120

16. Langer R, Diskin-Posner Y, Leitus G, Shimon LJ, Ben-David Y, Milstein D (2011) Angew Chem Int Ed 50:9948

17. Langer R, Iron MA, Konstantinovski L, Diskin-Posner Y, Leitus G, Ben-David Y, Milstein D (2012) Chem Eur J 18:7196

18. Zell T, Butschke B, Ben-David Y, Milstein D (2013) Chem Eur J 19:8068

19. Zell T, Ben-David Y, Milstein D (2014) Angew Chem Int Ed 53:4685

20. Zell T, Ben-David Y, Milstein D (2015) Catal Sci Technol 5:822

21. Berkessel A, Reichau S, von der Höh A, Leconte N, Neudörfl J-M (2011) Organometallics 30:3880

22. Sui-Seng C, Freutel F, Lough AJ, Morris RH (2008) Angew Chem Int Ed 47:940

23. Sui-Seng C, Haque FN, Hadzovic A, Pütz A-M, Reuss V, Meyer N, Lough AJ, Zimmer-De Iuliis M, Morris RH (2009) Inorg Chem 48:735

24. Lagaditis PO, Sues PE, Sonnenberg JF, Wan KY, Lough AJ, Morris RH (2014) J Am Chem Soc 136:1367

25. Zuo W, Tauer S, Prokopchuk DE, Morris RH (2014) Organometallics 33:5791

26. Tlili A, Schranck J, Neumann H, Beller M (2012) Chem Eur J $18: 15935$ 
27. Fleischer S, Zhou S, Junge K, Beller M (2013) Angew Chem Int Ed 52:5120

28. Benito-Garagorri D, Becker E, Wiedermann J, Lackner W, Pollak M, Mereiter K, Kisala J, Kirchner K (2006) Organometallics 25:1900

29. Benito-Garagorri D, Wiedermann J, Pollak M, Mereiter K, Kirchner K (2007) Organometallics 26:217

30. Benito-Garagorri D, Puchberger M, Mereiter K, Kirchner K (2008) Angew Chem Int Ed 47:9142

31. Benito-Garagorri D, Alves LG, Puchberger M, Mereiter K, Veiros LF, Calhorda MJ, Carvalho MD, Ferreira LP, Godinho M, Kirchner K (2009) Organometallics 28:6902

32. Benito-Garagorri D, Alves LG, Veiros LF, Standfest-Hauser CM, Tanaka S, Mereiter K, Kirchner K (2010) Organometallics 29:4923

33. Bichler B, Holzhacker C, Stöger B, Puchberger M, Veiros LF, Kirchner K (2013) Organometallics 32:4114

34. Bichler B, Glatz M, Stöger B, Mereiter K, Veiros LF, Kirchner K (2014) Dalton Trans 43:14517

35. Gorgas N, Stöger B, Pittenauer E, Allmaier G, Veiros LF, Kirchner K (2014) Organometallics 33:6905

36. Glatz M, Bichler B, Mastalir M, Stöger B, Mereiter K, Weil M, Pittenauer E, Allmaier G, Veiros LF, Kirchner K (2015) Dalton Trans 44:281

37. Holzhacker C, Stöger B, Carvalho MD, Ferreira LP, Pittenauer E, Allmaier G, Veiros LF, Realista S, Gil A, Calhorda MJ, Müller D, Kirchner K (2015) Dalton Trans 44:13071

38. Glatz M, Holzhacker C, Bichler B, Mastalir M, Stöger B, Mereiter K, Weil M, Veiros LF, Mösch-Zanetti NC, Kirchner K (2015) Eur J Inorg Chem 5053

39. Perrin DD, Armarego WLF (1988) Purification of laboratory chemicals, 3rd edn. Pergamon Press, New York

40. Junge K, Oehme G, Monsees A, Riermeier T, Dingerdissen U, Beller M (2003) J Organomet Chem 675:91

41. Bruker Computer Programs (2012) APEX2, SAINT, and SADABS. Bruker AXS Inc., Madison, WI

42. Palatinus L, Chapuis G (2007) J Appl Cryst 40:786

43. Petříček V, Dušek M, Palatinus L (2006) JANA2006, the crystallographic computing system. Institute of Physics, Praha, Czech Republic
44. Spek AL (2009) Acta Cryst D65:148

45. Macrae CF, Edgington PR, McCabe P, Pidcock E, Shields GP, Taylor R, Towler M, van de Streek J (2006) J Appl Cryst 39:453

46. Frisch MJ, Trucks GW, Schlegel HB, Scuseria GE, Robb MA, Cheeseman Jr, Scalmani G, Barone V, Mennucci B, Petersson GA, Nakatsuji H, Caricato M, Li X, Hratchian HP, Izmaylov AF, Bloino J, Zheng G, Sonnenberg JL, Hada M, Ehara M, Toyota K, Fukuda R, Hasegawa J, Ishida M, Nakajima T, Honda Y, Kitao O, Nakai H, Vreven T, Montgomery JA Jr, Peralta JE, Ogliaro F, Bearpark M, Heyd JJ, Brothers E, Kudin KN, Staroverov VN, Kobayashi R, Normand J, Raghavachari K, Rendell A, Burant JC, Iyengar SS, Tomasi J, Cossi M, Rega N, Millam JM, Klene M, Knox JE, Cross JB, Bakken V, Adamo C, Jaramillo J, Gomperts R, Stratmann RE, Yazyev O, Austin AJ, Cammi R, Pomelli C, Ochterski JW, Martin RL, Morokuma K, Zakrzewski VG, Voth GA, Salvador P, Dannenberg JJ, Dapprich S, Daniels AD, Farkas Ö, Foresman JB, Ortiz JV, Cioslowski J, Fox DJ (2009) Gaussian 09, Revision A.02. Gaussian Inc., Wallingford

47. Becke AD (1993) J Chem Phys 98:5648

48. Miehlich B, Savin A, Stoll H, Preuss H (1989) Chem Phys Lett 157:200

49. Lee C, Yang W, Parr G (1988) Phys Rev B 37:785

50. Haeusermann U, Dolg M, Stoll H, Preuss H (1993) Mol Phys 78:1211

51. Kuechle W, Dolg M, Stoll H, Preuss H (1994) J Chem Phys 100:7535

52. Leininger T, Nicklass A, Stoll H, Dolg M, Schwerdtfeger $P$ (1996) J Chem Phys 105:1052

53. McLean AD, Chandler GS (1980) J Chem Phys 72:5639

54. Krishnan R, Binkley JS, Seeger R, Pople JA (1980) J Chem Phys $72: 650$

55. Hay PJ (1977) J Chem Phys 66:4377

56. Raghavachari K, Trucks GW (1989) J Chem Phys 91:1062

57. Binning RC, Curtiss LA (1995) J Comput Chem 103:6104

58. McGrath MP, Radom L (1991) J Chem Phys 94:511 